\title{
Quantificação da ação do músculo frontal
}

\author{
Quantification of frontalis muscleaction
}

\author{
José Ribamar Souza da Cruz \\ Antonio Augusto Velasco e Cruz
}

\begin{tabular}{|l|}
\hline RESUMO \\
\hline Objetivo: Quantificar a ação do músculo frontal. Métodos: A ação do \\
músculo frontal foi quantificada por meio da medida da mobilidade do \\
supercílio em 81 sujeitos normais, divididos, segundo a faixa etária, em três \\
grupos: crianças com idades entre 6 e 12 anos $(\mathrm{n}=20)$, adultos com idades \\
entre 20 e 38 anos (n = 33) e idosos com idades entre 60 e 80 anos (n = 28). \\
As medidas foram realizadas com o processamento digital de imagem. \\
Obteve-se uma imagem da face do sujeito com o frontal em repouso e outra \\
com contração voluntária da fronte. Como índice de ação frontal foi tomada \\
a excursão do supercílio na parte média da fenda palpebral. Resultados: Os \\
valores médios da excursão superior do supercílio variaram, de acordo com \\
o grupo etário, de 7,57 a 9,02 mm. Apesar de nítida tendência de aumento \\
da mobilidade com a idade, as diferenças entre os três grupos não foram \\
significativas. Conclusões: A magnitude da excursão da porção central do \\
supercílio independe da idade.
\end{tabular}

Descritores: Músculos faciais/inervação; Fibras musculares; Músculos oculomotores; Pálpebras; Sobrancelhas; Contração muscular.

\section{INTRODUÇÃO}

A sigla SMAS (do inglês: Superficial Muscle-Aponeurotic System) é usada na literatura cirúrgica para designar o sistema fibromuscular superficial da face. Essa estrutura pode ser definida como uma camada fibromuscular que interconecta os músculos miméticos da face e a pele, sendo contínua com o platisma ${ }^{(1)}$. O conhecimento do SMAS é importante em cirurgia da face, pois toda a inervação motora para os músculos miméticos está subjacente a essa camada, de modo que a dissecção superior a este plano, acima do SMAS, é totalmente segura(2).

A espessura do SMAS é variável de uma região para outra da face. Na fossa temporal ele torna-se extremamente delgado sendo representado pela fáscia temporoparietal. Na região frontal, o SMAS é constituído pela gálea ${ }^{(3-4)}$.

Anatomicamente, a gálea aponeurótica ou, simplesmente, gálea é a estrutura fibrosa que une os dois ventres do músculo frontal, que é o músculo mimético do couro cabeludo. Em cirurgia, o termo gálea é usado comumente para designar o conjunto gálea aponeurótica mais músculo frontal. Esse fino músculo, de forma quadrangular, não apresenta inserções ósseas. Partindo de sua origem galeal, ele termina na pele e nos músculos adjacentes da região orbitária (orbicular, e corrugador) ${ }^{(5)}$.

$\mathrm{O}$ arranjo anatômico do músculo frontal permite uma alta mobilidade à região frontal. Os dois ventres do músculo occipitofrontal quando atuam conjuntamente, tracionam para trás o couro cabeludo, elevando os supercílios e enrugando a fronte como numa expressão de surpresa. Os ventres frontais podem agir isoladamente elevando somente os supercílios, de um ou de ambos os lados. 
Em várias situações clínicas a ação do músculo frontal está alterada, como por exemplo, nas paralisias periféricas de origem traumática ou viral, doença de Hansen, processos restritivos oriundos de cicatrizes, trauma cirúrgico e tumores. Todas essas condições em maior ou menor grau alteram a capacidade do músculo frontal de se contrair.

Não existem na literatura normas sobre a excursão do músculo frontal. O presente trabalho tem como objetivo quantificar a ação do músculo frontal, através da medida da mobilidade do supercílio em sujeitos normais de três faixas etárias: crianças, adultos jovens e idosos.

\section{MÉTODOS}

\section{População de estudo}

A população estudada compreendeu um grupo de 81 sujeitos normais divididos, segundo a faixa etária, em três grupos: grupo 1 - formado por crianças, com idades entre 6 a 12 anos, sendo 10 do sexo feminino e 10 do sexo masculino, grupo 2 constituído por 33 adultos jovens, na faixa etária de 20 a 38 anos, sendo 17 do sexo masculino e 16 do sexo feminino; grupo 2 - composto por 28 idosos de 60 a 80 anos de idade, sendo 13 do sexo masculino e 15 do sexo feminino. Para os três grupos os critérios de inclusão foram os mesmos: ausência de doenças sistêmicas ou oculares (no grupo idoso, a catarata não foi considerada como impeditiva), história negativa de trauma ou qualquer anomalia músculo-cutânea na região frontal.

\section{Método}

\section{1 - Aquisição das imagens}

Todos os indivíduos da população de estudo foram fotografados, de frente, com uma câmera digital. Os indivíduos eram sentados de maneira confortável e instruídos a olhar para frente (posição primária do olhar) com a cabeça mais ereta possível. Nessas condições, obtinha-se para cada sujeito duas imagens:uma com o frontal em repouso e a outra com contração voluntária máxima da fronte. Para essa segunda medida, solicitava-se ao sujeito que elevasse ao máximo os seus supercílios (Figuras 1 e 2).

\section{2 - Processamento digital e medidas}

Todas as imagens foram transferidas para um microcomputador Power Macintosh 8100. Apenas a imagem do olho direito foi processada pelo programa NIH 1.62 (escrito por Wayne Rasband do U.S National Institutes of Health e disponível pela internet no site http://rsb.info.nih.gov/nih-image/).

Após correção do contraste e eventuais rotações horizontais das imagens, foi traçada uma linha horizontal em toda extensão da fenda palpebral, no canto medial tomando a borda inferior da carúncula como reparo. Em seguida, uma linha vertical foi traçada a partir da linha de base horizontal, no intervalo médio entre os cantos medial e temporal (Figuras 1 e 2).

Todas as imagens foram parametrizadas (determinação da

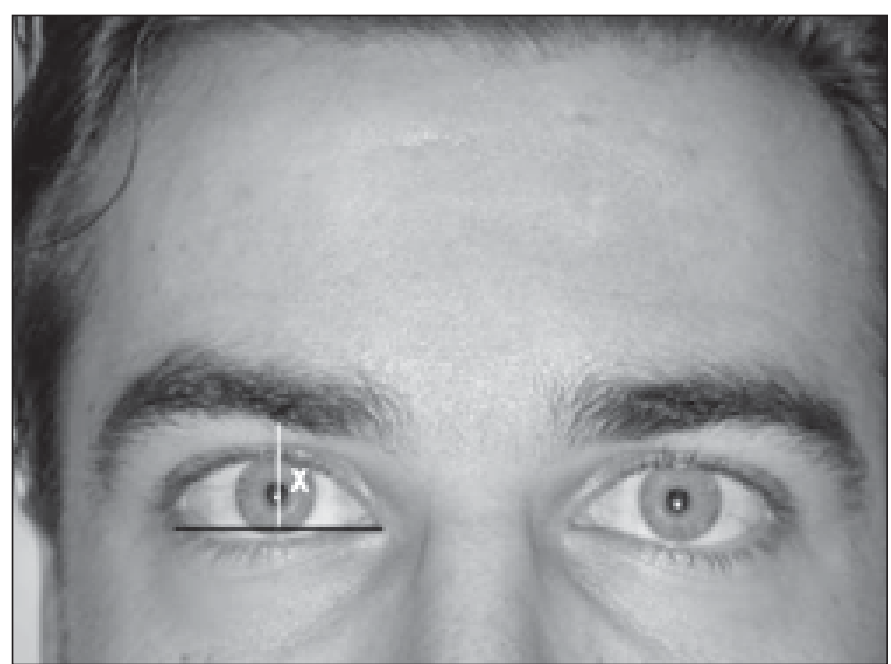

Figura 1 - Medida na posição de repouso da posição do supercílio (x)

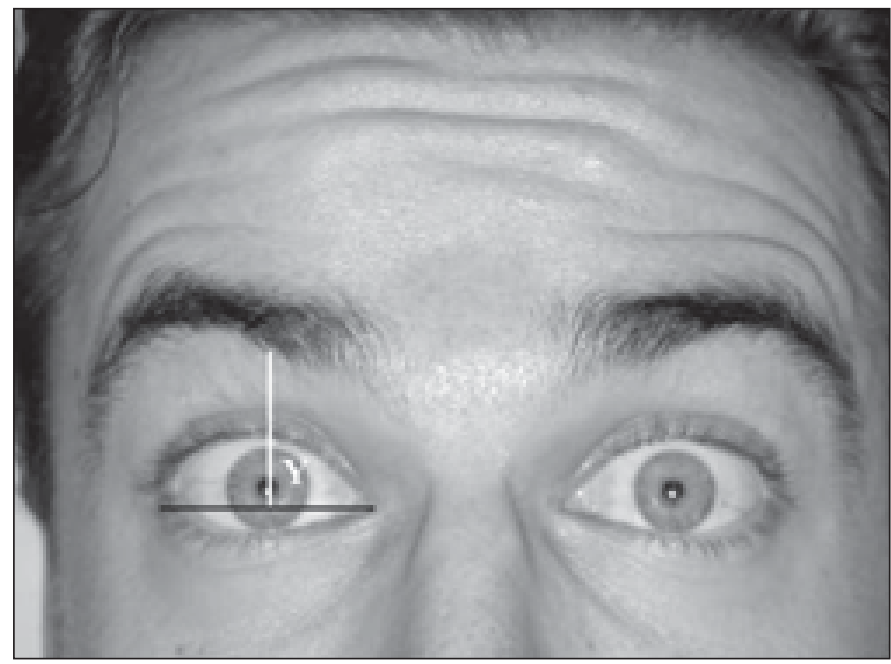

Figura 2 - Medida da posição do supercílio com contração frontal (y)

relação pixel/ milímetro) por meio de um detalhe de distância conhecida (diâmetro horizontal corneano $=12 \mathrm{~mm}$ ). A variável medida foi a excursão do supercílio (Y-X) tomada como índice do grau de ação do músculo frontal.

\section{Análise dos Dados}

Os valores da variável estudada foram comparados por meio de análise de variância unifatorial (ANOVA).

\section{RESULTADOS}

A tabela 1 relaciona os parâmetros das distribuições obtidas nos três grupos estudados.

Quando as médias foram comparadas por meio da ANOVA (Figura 3), verificou-se que não houve diferença estatisticamente significativa entre elas $(F=1,73, p=0,18)$. 


\begin{tabular}{|lccc|}
\hline \multicolumn{3}{|c|}{$\begin{array}{c}\text { Tabela 1 } \\
\text { Parâmetro }\end{array}$} \\
& Parâmetros das distribuições das excursões superci- \\
liares obtidas nos três grupos estudados & Grupo \\
Média & $\mathbf{1}$ & $\mathbf{2}$ & $\mathbf{3}$ \\
Mediana & 7,57 & 8,70 & 9,20 \\
Desvio-padrão & 6,39 & 8,43 & 9,58 \\
Erro-padrão & 3,47 & 2,59 & 3,14 \\
& 0,78 & 0,45 & 0,59 \\
\hline
\end{tabular}

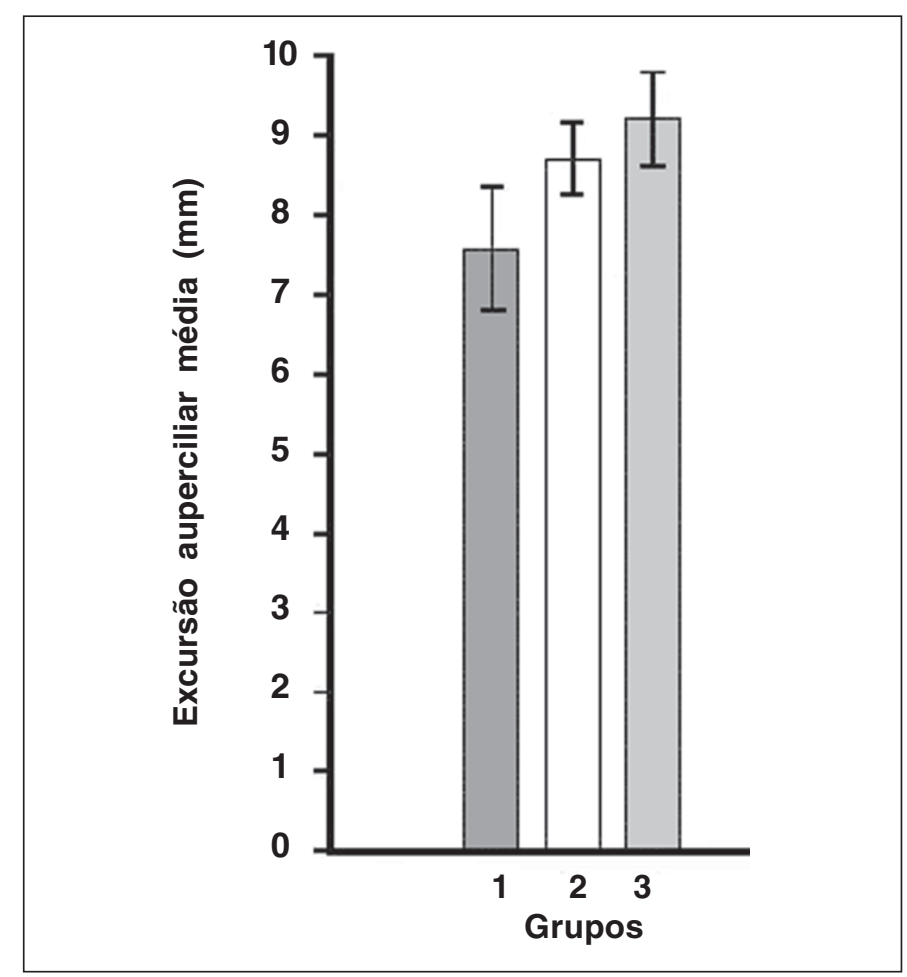

Figura 3 - Excursão superciliar média em função da faixa etária: 1 - crianças, 2 - adultos e 3 - idosos

\section{DISCUSSÃO}

A medida da função do músculo frontal pode ser importante em várias situações clínicas. A paresia ou paralisia induzida cirurgicamente durante a abordagem coronal é um dos principais problemas em cirurgia crânio-facial e cosmética da região frontal $^{(6-13)}$. Curiosamente, não há dados quantitativos sobre a excursão normal do músculo frontal.

Talvez, um dos protilemas relacionados à inexistência de dados a respeito da mag nitude da excursão do músculo frontal deva-se à dificuldade metodológica de se medir linhas e curvas, como as delineadas pelas margens superciliares. De fato, com instrumentos clínicos como réguas, compassos etc. é bastante difícil analisar-se quantitativamente linhas curvas e a mobilidade de estruturas anatômicas. O processamento computadorizado (digital) de imagens aplicado à cirurgia plástica ocular resolve este problema satisfatoriamente ${ }^{(14-20)}$.
No presente trabalho a excursão do músculo frontal, quantificada a partir da medida da mobilidade voluntária do supercílio, aumentou com a faixa etária. Uma possível explicação para a maior mobilidade do supercílio nos idosos seria decorrente do fato de a musculatura da mímica ser, teoricamente, mais empregada à medida que a idade progride. $\mathrm{O}$ frontal, principalmente, é contraído nos estados de surpresa e abaixado medialmente pelo corrugador durante os períodos de tensão e preocupação. A maior atividade muscular dos idosos é facilmente verificada pela profundidade e número de sulcos cutâneos na região frontal que aumentam com a idade.

Além disso, o SMAS é unido aos ossos da face e do crânio através de ligamentos fibrosos que limitam a sua mobilidade ${ }^{(3)}$. Na região orbitária esses ligamentos são especialmente importantes. No rebordo inferior há o denominado ligamento órbitomalar ${ }^{(21)}$. Na região lateral do supercílio, existe uma coalescência de fáscias na transição fronto-temporal. De fato, a fáscia temporal, a fáscia temporo-parietal e a própria gálea, fundem-se e são fixadas ao rebordo lateral por meio de um espesso ligamento lateral ${ }^{(22)}$. Esse ligamento é tão importante que os livros textos enfatizam a importância de sua liberação nas cirurgias de elevação do supercílio ${ }^{(23)}$. A explicação para a maior mobilidade do supercílio nos idosos poderia se dever à maior frouxidão ligamentar que naturalmente ocorre com a idade.

\section{CONCLUSÕES}

Os dados obtidos mostram que, apesar da mobilidade média ter aumentado com a faixa etária, essa diferença não foi estatisticamente significativa e, portanto, pode-se afirmar que, independentemente da idade, o supercílio é deslocado pela ação frontal na sua porção central em média de 7,5 a 9,2 $\mathrm{mm}$ em pessoas normais.

\section{ABSTRACT}

Purpose: To quantify the frontalis muscle action. Methods: The motion range of the eyebrow was measured in 81 normal subjects divided into three age groups: 20 children (ages from 6 to 12 years), 33 adults (ages from 20 to 38 years) and 28 elderly (ages from 60 to 80 years). Measurements were made using digital image processing analysis. For each subject an image of the subjects' face was obtained with the brow in its resting position. A second image was taken with maximal frontalis contraction. The frontalis action was taken as the difference between the position of the brow with and without frontalis contraction. All measurements were performed at halfway between the medial and lateral canthi. Results: Brow motility in the central part of the lid fissure ranges from 7.57 to $9.02 \mathrm{~mm}$. Despite a clear tendency to increase in brow motility with age, the differences between the age groups were not significant. Conclusions: The magnitude of the excursion of the central portion of the brow does not depend on age. 
Keywords: Facial muscles/innervation; Muscle contraction; Oculomotor muscles; Eyelids; Eyebrows

\section{REFERÊNCIAS}

1. Mitz V, Peyronie M. The superficial musculo-aponeurotic system (SMAS) in parotid and cheek area. Plast Reconstr Surg 1976;58:80-8.

2. Rudolph R. Depth of the facial nerve in face lift dissections. Plast Reconstr Surg 1990;85:537-44.

3. Mendelson BC. Surgery of the superficial musculoaponeurotic system: principles of release, vectors and fixation [commented on Plast Reconstr Surg 2002;109;824-5]. Plast Reconstr Surg 2001;107:1545-52; discussion p.1553-61.

4. Thaller SR, Kim S, Patterson H, Wildman M, Daniller A. The submuscular aponeurotic system(SMAS): a histologic and comparative anatomy evaluation [commented Plast Reconstr Surg 1991;88:545]. Plast Reconstr Surg 1990;86: 690-6.

5. Testut L. Muscles de la tête. In: Testut L, editor. Traité d'anatomie humaine. 7th ed. Paris: Librairie Octave Doin; 1921. p.715-48.

6. Yasargil MG, Reichman MV, Kubik S. Preservation of the frontotemporal branch of the facial nerve using the interfascial temporalis flap for pterional craniotomy. Technical article. J Neurosurg 1987;67:463-6.

7. Shepherd DE, Ward-Booth RP, Moos K. The morbidity of bicoronal flaps in maxillofacial surgery. Br J Oral Maxillofac Surg 1985;23:1-8.

8. Loeb R. Technique for preservation of the temporal branches of the facial nerve during face-lit operations. Br J Plast Surg 1970;23:390-4.

9. Connell BF, Lambros VS, Neurohr H. The forehead lift: techniques to avoid complications and produce optimal results. Aesthetic Plast Surg 1989;13: 217-37.

10. Liebman EP, Webster RC, Berger AS, Della Vecchia M. The frontalis nerve in the temporal brow lift. Arch Otolaryngol 1982;108:232-5.
11. Hochberg J, Kaufman H, Ardenghy M. Saving the frontal branch during a low fronto-orbital approach. Aesthetic Plast Surg 1995;19:161-3.

12. Gosain AK, Sewall SR, Yousif NJ. The temporal branch of the facial nerve: how reliably can we predict its path? Plast Reconstr Surg 1997;99:1224-33; discussion p.1234-5.

13. Baker DC, Conley J. Avoiding facial nerve injuries in rhytidectomy. Plast Reconstr Surg 1979;64:781-95.

14. Cruz AA, Coelho RP, Baccega A, Lucchezi MC, Souza AD, Ruiz EE. Digital image processing measurement of the upper eyelid contour in Graves disease and congenital blepharoptosis. Ophthalmology 1998;105:913-8.

15. Cruz AAV, Baccega A, Oliveira MVD, Malbouisson JM. A geometric model of eyelid contour [abstract]. Invest Ophthalmol Vis Sci 1999;40:29.

16. Cruz AAV, Luchezi MC. Quantification of palpebral fissure shape in severe congenital blepharoptosis. Ophthal Plast Reconstr Surg 1999;15:232-5.

17. Souza ADA, Ruiz EES, Cruz AAV. Palpebral fissure morphology segmentation and measurement using image processing. IEEE Eng Méd Biol Mag 2000; 19:114-9.

18. Cruz AAV, Baccega A. Análise bidimensional da fenda palpebral. Arq Bras Oftalmol 2001;64:13-9.

19. Paiva RS, Minaré-Filho AM, Cruz AA. Palpebral fissure changes in early childhood. J Pediatr Ophthalmol Strabismus 2001;38:219-23.

20. Hanada AL, Souza Jr EN, Moribe I, Cruz AAV. Comparison of palpebral fissure obliquity in three different racial groups. Ophthalmic Plast Reconstr Surg 2001;17:423-6.

21. Kikkawa DO, Lemke BN, Dortzbach RK. Relations of the superficial musculoaponeurotic system to the orbit and characterization of the orbitomalar ligament. Ophthal Plast Reconstr Surg 1996;12:77-88.

22. Knize DM. An anatomically based study of the mechanism of eyebrow ptosis. Plast Reconstr Surg 1996;97:1321-33.

23. Baker TJ, Gordon HL, Stuzin JM. Coronal browlifting. In: Baker TJ, Gordon HL, Stuzin JM, editors. Surgical rejuvenation of the face. 2nd ed. St. Louis: Mosby; 1996. p.528-73.

\title{
Jornada de Comemorag̣ão do XXXVIII Aniversário da Clínica Oeulisters Associados
}

\section{8 - 29 de Maio de 2004 Río do Janoiro - RJ}

\author{
INFORMAÇÕES: Tel.: (21) 2552-3522 \\ Fax: (21) 2553-5039
}

